\title{
A retrospective observational study on the sputum samples received by the Department of Microbiology, Teaching Hospital, Karapitiya in the year 2007
}

\author{
LU Edirisinghe ${ }^{1}$, Pankaja Kalukottage ${ }^{2}$, MNN Maziama $^{3}$, PV de Silva ${ }^{4}$ \\ ${ }^{I}$ Senior Registrar in Medicine, ${ }^{2}$ Consultant Microbiologist, Teaching Hospital, Karapitiya, Galle. \\ ${ }^{3}$ Consultant Chest Physician, Thassim Chest Clinic, Galle. \\ ${ }^{4}$ Lecturer in Community Medicine, Faculty of Medicine, University of Ruhuna, Galle. \\ e-mail address of the corresponding author,Dr.L.U.Edirisinghe: janpath100@gmail.com
}

\begin{abstract}
Background \& Objectives: The morbidity and mortality still remain significantly high in patients with severe lower respiratory tract infections. But data related to the offending organisms and their antibiotic sensitivity pattern is limited. Therefore, this study was designed to identify the bacterial pathogens and to review their antibiotic sensitivity by analyzing the results of sputum for culture samples which were received by Microbiology Department, Teaching Hospital, Karapitiya between January to December 2007.
\end{abstract}

Methods: All the sputum culture and antibiotic sensitivity test reports generated by the microbiology laboratory of the Teaching Hospital, Karapitiya from January to December 2007 were collected and analysed carefully in order to find out the organisms isolated and their antibiotic sensitivity pattern.

Results: Out of the 921 sputum culture reports generated by the microbiology laboratory in the year 2007, 322 had coliforms and 74 had Pseudomonas isolated. Furthermore, 16 isolates of coliforms (colony appearance resembled Klebsiella) and 13 isolates of Pseudomonas spp. were sensitive only to second line antibiotics.

Interpretation \& Conclusion: There were significant number of cases of Pseudomonas (18\%) and coliforms (5\%) (in which colony appearance resembled Klebsiella ) which were highly resistant to the first line antibiotics. In the severely ill patients with the clinical suspicion of above organisms, it is important to send sputum for culture and antibiotic sensitivity test and consider potent antibiotics such as imipenem, amikacin or netilmicin.

\section{Introduction}

Managing patients with lower respiratory tract infections is a challenge to a clinician as they account for a greater burden of disease worldwide than ischemic heart disease, cancer, malaria or human immunodeficiency virus infection ${ }^{1}$. The main guidelines on managing lower respiratory tract infections are laid down by British Thoracic Society $(\mathrm{BTS})^{2}$, American Thoracic Society (ATS ) ${ }^{3}$ and Infectious Disease Society of America (IDSA) ${ }^{4}$.

The most useful clinical classification of pneumonia is according to the possible origin of infection: community acquired, hospital acquired or pneumonia in immunocompromised patients ${ }^{5}$. Pathogenic organisms can be bacterial, viral, atypical or fungal and there is also considerable seasonal variation in some countries (eg: In the UK it is common during the winter) ${ }^{5}$.

A chest radiograph helps to confirm the diagnosis while sputum and blood cultures help to isolate the organism. Sputum cultures are recommended in severely ill patients with failure of antibiotic therapy or when there are pleural effusions or cavitary infiltrates ${ }^{4}$.

Latest guidelines laid by ATS / IDSA highlights the importance of identifying pathogenic organisms responsible for lower respiratory tract infections as it can significantly alter the standard (empirical) management decisions ${ }^{4}$. 
Previous studies have shown that Streptococcus pneumoniae (60-70\%) is the commonest offending organism in community acquired pneumonia followed by Haemophilus Influenzae (3-10\%) and Staphylococcus aureus (3-5\%)

But in our hospital set up, studies done on sputum culture isolates and their antibiotic sensitivity pattern are very much limited. So this study was planned to identify the common pathogenic organisms and their antibiotic sensitivity pattern among patients who got admitted to the Teaching Hospital, Karapitiya with lower respiratory tract infections.

\section{Materials and Methods}

Selection of request forms and worksheets.

All the sputum culture \& antibiotic sensitivity request forms sent to the microbiology laboratory, Teaching Hospital, Karapitiya from January to December 2007 were collected and analysed separately. Request forms in which the age was mentioned as below 12 years were not counted. In our laboratory, findings of sputum culture and antibiotic sensitivity pattern are usually documented on the request forms itself. These forms were used as worksheets in the laboratory and data maintained in the worksheets were analysed.

\section{Study Protocol:}

The study protocol was reviewed and approved by the Ethical Review Committee of Faculty of Medicine, University of Ruhuna. A specially prepared data sheet was used to record the findings.

\section{Reports Analysis:}

The specimens yielding significant pathogenic organisms were considered as positive cultures. Those which had scanty or insignificant growths were regarded as negative and when mixed growths of significant organisms were isolated, they were counted according to the predominant growth.
Statistical Analysis:

Data entry was completed using Microsoft Excel and Epinfo statistical package was used for data analysis. Chi square test was used to compare the populations.

\section{Results}

Table 1 illustrates the number of culture positive and negative sputum samples and the types of organisms isolated in each month. Out of the 920 culture reports analysed, significant pathogens were not isolated in 400 sputum samples. Out of the culture positive samples the commonest group of organisms isolated was coliforms spp. which account for 322 positive growths. Out of that, in 200 samples, the colony appearance closely resembled Klebsiella spp. and 30 of them were resistant to gentamicin. Out of the gentamicin resistant cases, $50 \%$ were sensitive to third generation cephalosporins and the rest were sensitive to second line antibiotics such as amikacin, netilmicin and imipenem.

There were 74 samples positive for Pseudomonas spp. out of which 13 were resistant to ceftazidime and were only sensitive to imipenem, meropenem or amikacin.

Staphylococcus aureus was isolated in 20 samples and 7 were Methicillin Resistant Staphylococcus aureus (MRSA) which were sensitive to Vancomycin.

Table 2 portraits the relationship between the age and the type of organism isolated. While coliforms were common among middle and elderly patients Pseudomonas spp. were almost equally distributed in young, middle and old age patients.

Figure 1 depicts the seasonal variations of coliforms and pseudomonas infections and a high incidence of both were recorded between May and August. 
Table 1 - Number of organisms isolated in each month

\begin{tabular}{|c|c|c|c|c|c|c|c|c|c|}
\hline $\begin{array}{l}\text { Coliform } \\
\text { spp. }\end{array}$ & $\begin{array}{l}\text { Pseudom } \\
\text { onas spp }\end{array}$ & $\begin{array}{l}\text { Pneumoc } \\
\text { occi }\end{array}$ & $\begin{array}{l}\text { Staphlo } \\
\text { coccus } \\
\text { aureus. }\end{array}$ & $\begin{array}{l}\text { Moraxella } \\
\text { catarrhalis } \\
\text { spp }\end{array}$ & $\begin{array}{l}\text { No } \\
\text { pathogn }\end{array}$ & $\begin{array}{l}\text { Candida } \\
\text { spp. }\end{array}$ & $\begin{array}{l}\text { Acinetoba } \\
\text { cters spp }\end{array}$ & $\begin{array}{l}\text { Proteus } \\
\text { spp }\end{array}$ & Total \\
\hline 15 & 4 & 4 & 3 & 6 & 30 & 2 & 1 & & 65 \\
\hline 20 & 2 & 1 & - & 4 & 65 & 1 & & & 93 \\
\hline 18 & 3 & 1 & 3 & 1 & 55 & 4 & & & 85 \\
\hline 28 & 7 & 1 & 2 & 4 & 33 & 3 & & & 78 \\
\hline 32 & 9 & - & - & 2 & 24 & 4 & & & 71 \\
\hline 39 & 10 & - & 3 & 10 & 37 & 5 & 4 & 1 & 109 \\
\hline 36 & 4 & 4 & & & 42 & 9 & & & 95 \\
\hline 37 & 6 & & 1 & 3 & 20 & & & & 67 \\
\hline 24 & 5 & 2 & 2 & 2 & 22 & & & & 57 \\
\hline 24 & 11 & 4 & 4 & & 20 & & & 1 & 64 \\
\hline 22 & 6 & 1 & 1 & 3 & 15 & 1 & & & 49 \\
\hline 27 & 7 & & 1 & 2 & 37 & 13 & 1 & & 88 \\
\hline 322 & 74 & 18 & 20 & 37 & 400 & 42 & 6 & 2 & 921 \\
\hline
\end{tabular}

Table 2 -Age and the organisms isolated.

\begin{tabular}{|c|c|c|}
\hline Age & $\begin{array}{l}\text { Number of } \\
\text { coliform isolates }\end{array}$ & $\begin{array}{l}\text { Number of Pseudomonas } \\
\text { isolates. }\end{array}$ \\
\hline $15-40$ & 55 & 21 \\
\hline $41-60$ & 92 & 22 \\
\hline $61-90$ & 142 & 20 \\
\hline
\end{tabular}

Figure 1 - Seasonal variations of infection

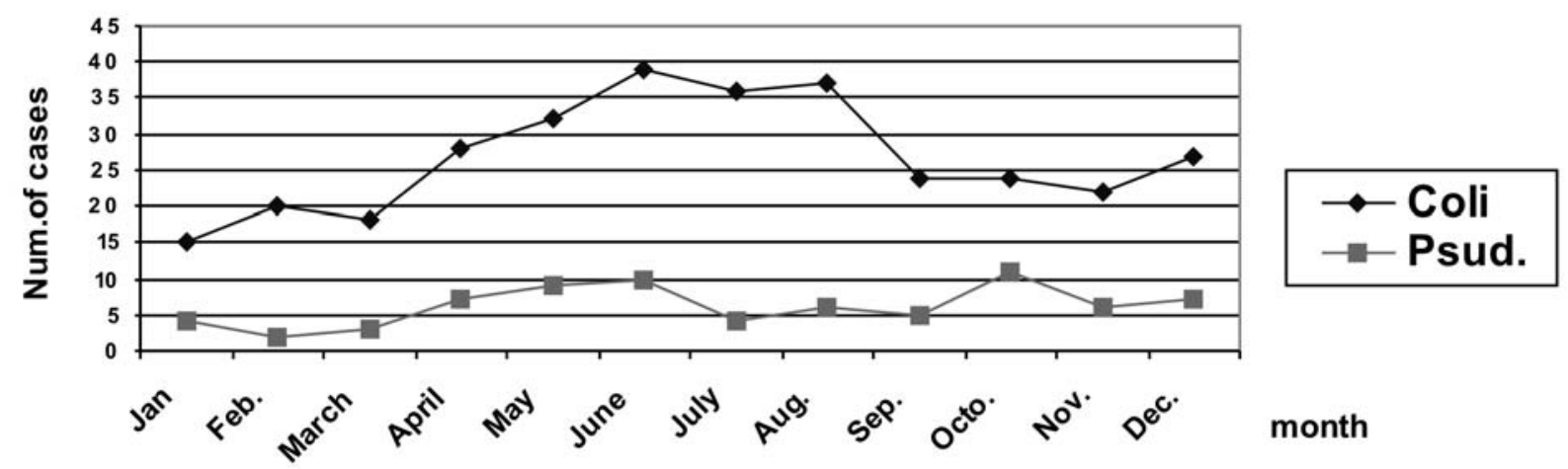




\section{Discussion}

It is well known that Streptococcus pneumoniae (Pneumococcus) is the commonest causative organism (35\%) for community acquired pneumonia ${ }^{5}$. Furthermore, it is common among the elderly and a high incidence is found during the winter seasons ${ }^{5}$.

But the yield of $S$. pneumoniae from the sputum cultures of patients with bacteremic pneumococcal pneumonia is only $40 \%-50 \%$, according to the studies conducted a few decades ago $^{8,9}$.

In this study Pneumococci were isolated only in 18 sputum samples. This may be due to the sensitivity of this organism to antibiotics and most patients may have had antibiotics prior to hospital admission. Out of the 921 culture reports analysed, 400 sputum samples were negative for pathogens. This could be due to partially treated patients before or during the hospital stay, or these patients may be affected by viral, fungal or atypical pathogens (Table 1).

Furthermore, the results of sputum bacterial cultures are strongly influenced by specimen collection, transport, rapid processing, satisfactory use of cytologic criteria, and the skill in interpretation ${ }^{4}$.

In this study we have found that coliforms were isolated in a relatively large proportion (34\%) of sputum samples. In fact, it was the commonest type of pathogenic organism isolated in sputum cultures (Table1). In a considerable number of samples $(62 \%)$ the colony appearance of coliforms resembled Klebsiella spp, but lack of facilities to accurately identify this organism in our hospital was a major drawback of this study. Moreover, in some samples where colony appearance resembled Klebsiella spp. the radiological findings also correlate with it. (Typical upper lobe involvement). Some patients had complications like haemoptysis, plural effusions, lung abscess formation or poorly resolving pneumonia. Even though nearly $90 \%$ of the isolates were highly sensitive to first line antibiotics like gentamicin or ceftazidime, $6 \%$ of cases were highly resistant to the first line antibiotics and were sensitive only to more expensive second line antibiotics like imipenem, meropenem, amikacin or netilmicin. In patients with severe pneumonia where Klebsiella is suspected on clinical and radiological grounds with the patients responding poorly to the current treatment regimen, one has to be very vigilant of it's resistance to first line antibiotics.

Pseudomonas was the second commonest organism isolated from sputum cultures. Out of the 74 positive cases, 13 were resistant to ceftazidime and were only sensitive to potent antibiotics such as amikacin, imipenem or meropenem.

According to the study findings, 7 cases of MRSA were identified out of the 20 Staphylococcus aureus isolates. All those isolates were sensitive to vancomycin. So in cases of Staphylococcal pneumonia, clinicians have to be aware of this possibility. especially if the patient is not responding to the current treatment.

Unlike the Pseudomonas isolates, which were equally distributed among young, middle and old age group patients, coliforms were commonly isolated from middle and old age groups (Table 2). Statistical analysis further confirmed that there is a statistically significant difference between coliform and Pseudomonas infections with the age. Previous studies done in the UK also revealed that the number of cases of pneumonia rapidly starts to rise after the age of 70 years ${ }^{7}$.

A seasonal variation is also identified in the incidence of lower respiratory tract infections in the UK. During the winter the number of cases started shooting up. In our study also when we analysed the incidence of Coliforms and Pseudomonas spp. cases each month, there was a clear seasonal variation. Lower respiratory tract infections caused by both organisms were increasingly detected during May, June and July which correlate with South- East monsoon rains in this part of the island (Figure 1).

\section{Conclusion}

This study revealed that coliforms were the commonest isolated organism from the sputum samples of patients suffering from lower respiratory tract infections. There was a significantly high proportion of patients who were affected by drug resistant strains. 


\section{Recommendation}

Before starting empirical antibiotics on patients with moderate to severe lower respiratory tract infections, it is always advisable to obtain sputum for culture and ABST.

\section{Acknowledgements}

We gratefully acknowledge Dr. Ajith Nagahawatte in the Department of Microbiology, Faculty of Medicine, University of Ruhuna and Dr. (Mrs.) D. Vidanagama in the Department of Microbiology, Teaching Hospital, Karapitiya for their kind assistance in carrying out this research.

\section{References}

1. Joseph P, Mizgerd SD. Acute Lower Respiratory Tract Infection. The New England Journal of Medicine 2008; 358: 716-24.

2. British Thoracic Society. Guidelines for the management of community-acquired pneumonia in adults. Thorax 2001; 56(Suppl. IV): 1-56.
3. American Thoracic Society. Hospital-acquired pneumonia in adults: diagnosis, assessment of severity, initial antimicrobial therapy, and preventative strategies. Am J Respir Crit Care Med 1995; 153: 1711-25.

4. IDSA / ATS Guidelines for CAP in Adults. Clinical Infectious Diseases 2007; 44: S27-72.

5. Wilkinson M, Woodhead M. Pneumonia. Medicine International 2004; 32(2): 129-34.

6. Mclnotsh K. Community - acquired pneumonia in children. NEnglJMed 2002; 346: 429-37.

7. Bartlett GW, Mundy LM. Community - acquired pneumonia. NEnglJMed 1995; 333: 1618-24.

8. Barrett-Connor E. The non-value of sputum culture in the diagnosis of pneumococcal pneumonia. Am Rev Respir Dis 1971; 103: 845-8.

9. Lentino JR, Lucks DA. Non-value of sputum culture in the management of lower respiratory tract infections. J Clin Microbiol 1987; 25: 758-62. 